\title{
Polyphenols extraction from plant sources
}

\author{
Aleksandra Jovanović ${ }^{1,}$, Predrag Petrović $^{1}$, Verica Đorøević ${ }^{1}$, Gordana Zdunić ${ }^{2}$, \\ KATARINA ŠAVIKIN ${ }^{2}$, AND BRANKO BUGARSKI ${ }^{1}$ \\ ${ }^{1}$ Faculty of Technology and Metallurgy, University of Belgrade, Karnegijeva 4, 11000 Belgrade, Serbia \\ ${ }^{2}$ Institute for Medicinal Plant Research "Dr. Josif Pančić", Tadeuša Košćuška 1, 11000 Belgrade, Serbia \\ *Corresponding author: acancarevic@tmf.bg.ac.rs
}

Received: October 11, 2017

Accepted: November 11, 2017

Published on-line: November 13, 2017

Published: December 25, 2017

\begin{abstract}
Different conventional (maceration and heat-assisted extraction) and new alternative techniques (ultrasound-assisted and microwave-assisted extractions) have been developed for the extraction of polyphenols compounds from different plant sources. Novel procedures have established with the aim to reduce the extraction time and solvent consumption, as well as to increase polyphenols yield and to improve extract quality. A critical review was conducted to introduce and compare traditional and modern procedures applied for extraction of bioactive polyphenols compounds. This review focuses on the different techniques of polyphenols extraction, discussing their operating conditions, mechanism, choice of particle size and solvent, solid/solvent ratio, extraction time, advantages/disadvantages and effectiveness. Finally, potential application of these extraction procedures in polyphenols isolation is reviewed.
\end{abstract}

Key words: extraction, polyphenols, particle size, solvents, solid/solvent ratio, time

http://dx.doi.org/10.5937/leksir1737045」

\section{INTRODUCTION}

Polyphenols represent a wide group of plant secondary metabolites, which possess numerous biological activities including antioxidant, antimicrobial, antispasmodic, antiinflammatory, antiallergic, hepatoprotective and anticarcinogenic properties (Ben El Hadj Ali et al., 2014; Bhatt et al., 2017; Munin and Edwards-Levy, 2011; Sen and Chakraborty, 2011). Therefore, there is a growing interest for isolation of these phytochemicals from plant materials, with the aim to produce a safe, natural and low-cost alternative to synthetic compounds (Ben El Hadj Ali et al., 2014; Canadanović-Brunet et al., 2006). Generally, liquid and dried plant extracts (rich in active constituents) are widely used in food, pharmaceutical and cosmetic industries (Wang and Weller, 2006). Considering that polyphenols from different plants vary widely in their structure, it is not possible to establish a standard extraction protocol that would extract all targeted compounds from each plant source (Bucić-Kojić et al., 2011). In the recent studies, different procedures for polyphenols extraction were developed and they vary in mechanism, nature of plant material, solvent type, solid/solvent ratio, time, temperature, pressure and solvent pH (Ben El Hadj Ali et al., 2014; Bucić-Kojić et al., 2011; Cam and Hisil, 2010; Galvan d'Alessandro et al., 2012). Traditional procedures for obtaining plant extracts include maceration, percolation, digestion and Soxhlet extraction (Vuleta et al., 2012; Wang and Weller, 2006). These methods are simple, but involve several disadvantages such as low polyphenols yield, long extraction time and large amounts of plant material and solvent (Mustafa and Turner, 2011; Wang and Weller, 2006). In the recent time, the application of novel extraction procedures, such as ultrasound-assisted, microwave-assisted, supercritical fluid, accelerated solvent and pressurized liquid extractions, have been evaluated (Both et al., 2014; Dahmoune et al., 2015; Milošević et al., 2011; Mustafa and Turner, 2011; Oniszczuk and Podgorski, 2015). According to the literature data, these methods provide various benefits which include higher yield of polyphenols, shorter extraction time, ie. faster kinetics, reduction of organic solvent consumption and positive environmental impact (Wang and Weller, 2006).

This review focuses on the different techniques of polyphenols extraction (maceration, heat-assisted, ultrasound-assisted and microwave-assisted extractions), discussing their operating conditions, mechanism, choice of particle size and solvent, solid/solvent ratio, extraction time, advantages/disadvantages and effectiveness. Finally, potential application of those extraction procedures for polyphenols isolation is reviewed.

\section{EXTRACTION TECHNIQUES}

\section{Maceration}

Maceration is a traditional, well-established and simple procedure of polyphenols extraction from plant sources. In terms of operating conditions, maceration is performed at room temperature in glass containers with continuous mixing during 
10-30 minutes or several hours, depending on the characteristics of plant matrix. The extraction is carried out according to the principle of polyphenols diffusion into an appropriate extraction medium (Vuleta et al., 2012). Maceration strongly depends on the properties of plant material, particularly particle size, thus the internal diffusion may be the limiting step during extraction (Wang and Weller, 2006). According to the literature, the highest polyphenols yield from Aronia melanocarpa and Thymus serpyllum is achieved with the smallest particles, because of the increased active surface area and the enhanced contact of matrix with solvent (Ćujic et al., 2016; Jovanović et al., 2017). The choice of solvent depends on physical and chemical properties of target compounds, as well as on applied extraction procedure (Wang and Weller, 2006). Since that polyphenols extracts are commonly used for food, medicals and cosmetics, the choice of solvent represents a critical step. Water, ethanol, methanol and their mixture are the most commonly applied solvents for polyphenols extraction (Aziz and Habib-Ur-Rehman, 2008; Cujic et al., 2016; Vajić et al., 2015). According to the recent studies, polyphenols from aromatic plants are preferentially extracted with mixture of alcohol and water using maceration, particularly when they are in a glycoside form (Costa et al., 2012; Jovanović et al., 2017; Vajić et al., 2015). In a mixture, one solvent can enhance the solubility of the polyphenols, while the other can improve desorption (Mustafa and Turner, 2011). Moreover, the small quantity of water in binary solvent system has significant impact, because it creates a more polar medium, whereas breaking hydrogen bonding facilitates the extraction of polyphenols (Uma et al., 2010). In maceration, the increase of solid/solvent ratio leads to the increase of polyphenols yield, which can be explained by the prevention of saturation of extraction medium using higher solvent volume (Bucić-Kojić et al., 2007; Ćujic et al., 2016). One of the disadvantages of maceration as extraction technique is a need for a great amount of solvent, in order to achieve better extraction yield. On the other hand, the presence of a large amount of plant material contributes to the increase of extraction medium viscosity, which causes the reduction of diffusion rate (Vuleta et al., 2012). According to Fick's second law of diffusion, by prolongation of the extraction time, the polyphenols yield is increased (Nayak et al., 2015). However, in the polyphenols extraction from $A$. melanocarpa, T. serpyllum, Urtica dioica and Lawsonia inermis at room temperature, the extraction time did not have statistically significant influence on polyphenols yield (Uma et al., 2010; Cujic et al., 2016; Jovanović et al., 2017; Vajić et al., 2015). The reason could be in occurrence of two stages in maceration: an initial increase of the polyphenols content in the beginning of the process, followed by slow extraction (Vuleta et al., 2012). The advantages of maceration: (1) particularly convenient for thermosensitive components, (2) using various plant materials, solvents and $\mathrm{pH}$, and (3) simple and cheap method. The main disadvantages of maceration include (1) long extraction time, (2) a large quantity of solvent, and (3) lower polyphenols yield. Maceration is widely and successfully applied procedure for extraction of thermosensitive polyphenols compounds from different plant sources (Ćujic et al., 2016; Jovanović et al., 2017; Vajić et al., 2015). Nevertheless, in comparison to novel extraction methods, maceration is old-fashion and time-consuming extraction procedure, which does not provide high polyphenols yield (Jovanović et al., 2017).

\section{Heat-assisted extraction}

Heat-assisted extraction is carried out at high temperature in glass containers with continuous mixing in water bath or in the incubator shaker, allowing unattended operation in a temperature-controlled environment. The use of thermal energy improves the efficiency of the extraction by disruption of cellular structures, increment of cell membrane permeability and breakdown of polyphenols-lipoproteins interactions, which cause enhancement of polyphenols solubility and mass transfer (Mustafa and Turner, 2011). High temperature decreases viscosity of the extraction medium, which helps the solvent to penetrate the plant matrix, and results in faster kinetics (Miron et al., 2011). According to Vergara-Salinas et al. (2012), the increment of solvent temperature decreases surface tension and, consequently, enhances wetting of the plant particles, leading to higher extraction yield. In the extraction at high temperature, the decrease in particle size of Vitis vinifera seeds, skins and stems causes the increase of polyphenols yield (Bucić-Kojić et al., 2007; Pinelo et al., 2005). In heat-assisted extraction, the maximum polyphenols content from Ficus carica, Origanum vulgare, Artemisia dracunculus and T. serpyllum was obtained using a mixture of ethanol and water (Bucić-Kojić et al., 2011; Miron et al., 2011). According to the literature, the polarity of solvent decreases by applying high temperature during the extraction, which makes water and ethanol suitable solvents to extract polar, moderately polar and non-polar organic constituents (Mustafa and Turner, 2011). Due to very low polyphenols yield proven in several previous studies, mono-solvent system is not recommended as an appropriate solvent for polyphenols extraction at high temperature (Chizzola et al., 2008; Jovanović et al., 2017; Miron et al., 2011). Several studies have revealed that solid/solvent ratio and temperature had significant influence on polyphenols yield and with the increase of ratio the polyphenols content in extracts also increases (Bucić-Kojić et al., 2007; Jovanović et al., 2017; Pompeu et al., 2009). According to Fecka and Turek (2008), in hot water and methanol extracts of T. serpyllum and T. vulgaris, the extraction time did not significantly affect polyphenols content. Additionally, the exposure time had no statistically significant influence on polyphenols extraction from wild sage and thyme (Vergara-Salinas et al., 2012; Dent et al., 2013). Moreover, both high temperature and long exposure time can reduce the extraction yield, because of temperature sensitivity and enzymatic degradation and oxidation of polyphenols, as well as polymerization among insoluble constituents (Vergara-Salinas et al., 2012). The main advantages of heat-assisted extraction include (1) the enhancement of polyphenols solubility and mass transfer caused by high temperature, (2) faster kinetics, (3) the efficient extraction and higher polyphenols yield, and (4) simple apparatus and method. The disadvantages of heat-assisted extraction: (1) degradation of thermosensitive polyphenols compounds, (2) high solvent consumption, and (3) limited choice of plant material and extraction medium. According to the results of previous studies, heat-assisted extraction is widely and successfully used extraction technique to obtain polyphenols extracts from different plants, because high temperature has a positive influence on the efficiency of polyphenols extraction, particularly from aromatic plants of Lamiaceae family, such as sage, rosemary, marjoram and oregano (Hossain et al., 2011; Miron et al., 2011; Dent et al., 2013). In comparison to maceration and taking into consideration the industrial requirements such as maximum polyphenols yield for a shorter time, heat-assisted extraction represents convenient technique for polyphenols extraction from various plant sources (Hossain et al., 2011; Miron et al., 2011).

\section{Ultrasound-assisted extraction}

Ultrasound-assisted extraction can be performed using ultrasound bath or ultrasound probe. The mechanism of ultrasound-assisted extraction include plant tissue destruction by ultrasound waves, which travel inside of plant cells as mechanical vibrations and cause expansion and compression cycles during movement through the extraction medium 
and provoke local rise of temperature and negative pressure (Wang and Weller, 2006). These mechanical and thermal effects cause the degradation of cell walls, release of cell contents, a greater penetration of solvent into plant material, the increment of mass transfer and thus, the increase of polyphenols yield (Deng et al., 2015; Horžic et al., 2012). Particle size of plant material does not significantly affect polyphenols yield, since ultrasound waves induce reduction of the particle size (approximately 5\%), damage of the cells and modification of microstructure, which results in the increase of solvent penetration into herbal matrix and in the higher polyphenols release, regardless of the initial size of plant particles (Both et al., 2014; Deng et al., 2015; Jovanović et al., 2017). Regarding the selection of the extraction medium for ultrasound-assisted extraction, alcohol/water mixture was the most suitable solvent for maximum recovery of polyphenols from Jatropha dioica, Eucalyptus camaldulensis, Origanum majorana and Thymus species, whereas pure alcohol or water could not completely extract polyphenols (Chizzola et al., 2008; Fecka and Turek, 2008; Wong Paz et al., 2015). According to the results of previous publications, polyphenols content in plant extracts obtained in ultrasound-assisted extraction was higher as solid/solvent ratio increased (Jovanović et al., 2017; Yang and Zhang, 2008; Wong Paz et al., 2015). This could be explained by the mechanism of ultrasound waves which generate changes in the plant material, enhance the mass transfer rate, and thus faster saturation of the liquid. Therefore, the increase in solid/solvent ratio leads to the prevention of saturation of the extraction medium and the increment of polyphenols content in the extracts (Wong Paz et al., 2015). On the other hand, the presence of a large amount of plant matrix contributes to the attenuation of the ultrasonic waves and the active part is restricted to a small zone (Wang and Weller, 2006). The extraction time did not have statistically significant influence on polyphenols yield in ultrasound-assisted extraction from stinging nettle leaf and wild thyme herb (Jovanović et al., 2017; Vajić et al., 2015). Additionally, the extended time can damage extracted natural antioxidants and degrade extract quality, due to higher temperature and free radicals produced by the ultrasound waves (Horžic et al., 2012). The advantages of ultrasound-assisted extraction include (1) the increase of extraction yield, (2) the improvement of the extract quality, (3) fast kinetics - the important property for industrial requirements, (4) the ultrasound instrument has lower price and it is easier to work on it, in comparison to other modern extraction techniques, and (5) a wide range of solvents can be used for extraction of different pharmacologically and biologically active phytochemicals. The disadvantages of ultrasound-assisted extraction: (1) degradation of antioxidant polyphenols compounds, (2) the effects of ultrasound waves on polyphenols yield and extraction kinetics depend on the characteristics of plant material, and (3) the presence of a larger quantity of plant particles contributes to the ultrasound waves attenuation, which results in the restriction of the active part of ultrasound inside the zone located in the vicinity of the ultrasonic emitter. Very high yield of polyphenols in extracts obtained using ultrasound-assisted extraction and shorter extraction time, compared to traditional extraction techniques, were demonstrated in several studies (Hossain et al., 2011; Khan et al., 2010; Horžic et al., 2012; Lee et al., 2013). Therefore, ultrasound-assisted extraction can be proposed as an efficient and fast procedure for extraction of bioactive polyphenols from different plant materials (Vajić et al., 2015; Wang and Weller, 2006; Horžic et al., 2012).

\section{Microwave-assisted extraction}

Modern techniques, such a microwave-assisted extraction, have been applied as an alternative to the traditional proce- dures for polyphenols extraction, with the aim to improve the extraction efficiency, as well as to provide shorter extraction time, lower solvent consumption and higher polyphenols yield. Microwave-assisted extraction can be carried out using microwave oven or microwave reactor (which ensures better control of temperature and pressure in the samples). Microwaves offer a rapid delivery of energy to a total volume of extraction medium and plant particles, which leads to the efficient and homogeneous heating of the sample. Moreover, water within the plant cells absorbs microwave energy and internal superheating causes cell disruption, which result in the improvement of the polyphenols recovery (Wang and Weller, 2006). According to Chupin et al. (2015), in the microwave-assisted extraction of Pinus pinaster, in terms of polyphenols content, there was no statistically significant difference between particles size of $0.4-1 \mathrm{~mm}$, whereas the highest content was obtained using the smallest particles, $<0.1 \mathrm{~mm}$. Apart from that, the best efficiency of microwave-assisted extraction of polyphenols from Hippophae rhamnoides and $A$. melanocarpa was achieved with a degree of fragmentation less than $0.5 \mathrm{~mm}$ (Asofiei et al., 2016; Dandena et al., 2014). According to the recent publications, the increase of ethanol concentration in water medium caused the increase in the polyphenols yield from Achillea millefolium and Myrtus communis, which can be explained by polar distribution and dielectric constant (Dahmoune et al., 2015; Milutinović et al., 2015). The increase in dielectric constant, which is a result of the addition of water to ethanol, allows the absorption of microwaves energy. However, higher quantities of water lead to a reduced expanding of heat into extraction medium, which also reduces the efficiency of microwave extraction (Milutinović et al., 2015). Additionally, the highest content of polyphenols from $A$. melanocarpa was achieved by using $50 \%$ ethanol, whereas further increase of ethanol concentration led to the decrease of polyphenols yield (Simić et al., 2016). Milutinović et al. (2015) have shown that in microwave-assisted extraction, the increase in solid/solvent ratio resulted in the enhancement of polyphenols content from A. millefolium, due to more efficient wetting of plant material. The increase in the diffusion coefficient and the intensive release of polyphenols from destroyed plant cells caused by microwaves, lead to the rapid saturation of the extraction medium. Therefore, the increase of solvent volume provides better polyphenols recovery from plant material (Wang and Weller, 2006; Chupin et al., 2015). According to the literature data, high power of microwaves during short extraction time can ensure the degradation of cell wall and better diffusion of polyphenols into extraction medium. Apart from that, longer time and better yields of microwave extractions do not mean a large quantity of target compounds, because microwaves cause the release of a large amount of ballast substances, such as lipids, proteins and polysaccharides (Simić et al., 2016). The main advantages of microwave-assisted extraction: (1) significant reduction of extraction time, (2) reduction of solvent consumption, (3) increased extraction yield, and (4) simplicity and economy of the extraction process, in comparison to other novel extraction procedures, such as supercritical fluid extraction. The disadvantages of microwave-assisted extraction include (1) degradation of thermosensitive polyphenols constituents, (2) lower yield of non-polar and volatile target compounds, (3) the effects of microwaves on polyphenols yield depend on the polarity of solvents, and (4) a possibility of the extraction of a large quantity of ballast substances. Microwave-assisted extraction is widely and successfully applied technique for obtaining polyphenols extracts from different plant matrixes, such as chokeberry fruit and yarrow, myrtle and sea buckthorn leaves (Asofiei et al., 2016; Milutinović et al., 2015; Simić et al., 2016; Dandena et al., 2014). From the point of view of 
environmental protection and food, pharmaceutical and cosmetic industries, which require safe and high quality products, with the application of "green" extraction methods, which are quick and automated, microwave-assisted extraction is a recommended method for obtaining high quality extracts from numerous plant sources, with high polyphenols yield (Bouras et al., 2015).

\section{CONCLUSION}

There is a growing interest in extracting polyphenols from plant sources, in order to produce a safe, natural and lowcost alternative to synthetic compounds, out of which some possess toxic and mutagenic effects. Conventional extraction procedures, such as maceration and heat-assisted extraction, as simple methods, can be applied for polyphenols extraction from different plant materials, but their main disadvantage is high solvent consumption, which increases operating costs and causes additional environmental problems, whereas polyphenols yield is lower, in comparison to novel extraction techniques. Several modern extraction methods, such as ultrasound-assisted extraction and microwave-assisted extraction, have been established, offering the advantages with respect to extraction time, solvent consumption and polyphenols yield. From the point of view of environmental protection and food, pharmaceutical and cosmetic industries, which require the application of "green" extraction procedures, ultrasoundassisted extraction and microwave-assisted extraction are recommended to obtain extracts rich in polyphenols from various plant sources.

\section{REFERENCES}

Asofiei, I., Calinescu, I., Trifan, A., David, I. G. and Gavrila, A. I. (2016). Microwave-Assisted Batch Extraction of Polyphenols from Sea Buckthorn Leaves, Chemical Engineering Communications 203(12): 1547-1553.

Aziz, S. and Habib-Ur-Rehman (2008). Studies on the Chemical Constituents of Thymus serpyllum, Turkish Journal of Chemistry 32: 605-614.

Ben El Hadj Ali, I., Bahri, R., Chaouachi, M., Boussaid, M. and Harzallah-Skhiri, F. (2014). Phenolic content, antioxidant and allelopathic activities of various extracts of Thymus numidicus Poir. organs, Industrial Crops and Products 62: 188195.

Bhatt, V., Sharma, S., Kumar, N., Sharma, U. and Singh, B. (2017). Simultaneous quantification and identification of flavonoids, lignans, coumarin and amides in leaves of Zanthoxylum armatum using UPLC-DAD-ESI-QTOF-MS/MS, Journal of Pharmaceutical and Biomedical Analysis 132: 46-55.

Both, S., Chemat, F. and Strube, J. (2014). Extraction of polyphenols from black tea - Conventional and ultrasound assisted extraction, Ultrasonics Sonochemistry 21(3): 1030-1034.

Bouras, M., Chadni, M., Barba, F. J., Grimi, N., Bals, O. and Vorobiev, E. (2015). Optimization of microwave-assisted extraction of polyphenols from Quercus bark, Industrial Crops and Products 77: 590-601.

Bucić-Kojić, A., Planinic, M., Tomas, S., Bilić, M. and Velić, D. (2007). Study of solid-liquid extraction kinetics of total polyphenols from grape seeds, Journal of Food Engineering 81(1): 236-242.

Bucić-Kojić, A., Planinic, M., Tomas, S., Jokic, S., Mujic, I., Bilic, M. and Velic, D. (2011). Effect of Extraction Conditions on the Extractability of Phenolic Compounds from
Lyophilised Fig Fruits (Ficus Carica L.), Polish Journal of Food and Nutrition Sciences 61(3).

Cam, M. and Hisil, Y. (2010). Pressurised water extraction of polyphenols from pomegranate peels, Food Chemistry 123(3): 878-885.

Canadanović-Brunet, J. M., Đilas, S. M., Ćetkovic, G. S., Tumbas, V. T., Mandić, A. I. and Čanadanovic, V. M. (2006) Antioxidant activities of different Teucrium montanum L. Extracts, International Journal of Food Science and Technology 41(6): 667-673.

Chizzola, R., Michitsch, H. and Franz, C. (2008). Antioxidative Properties of Thymus vulgaris Leaves: Comparison of Different Extracts and Essential Oil Chemotypes, Journal of Agricultural and Food Chemistry 56(16): 6897-6904.

Chupin, L., Maunu, S., Reynaud, S., Pizzi, A., Charrier, B. and Charrier-EL Bouhtoury, F. (2015). Microwave assisted extraction of maritime pine ( Pinus pinaster ) bark: Impact of particle size and characterization, Industrial Crops and Products 65: 142-149.

Costa, P., Goncalves, S., Valentao, P., Andrade, P. B., Coelho, N. and Romano, A. (2012). Thymus lotocephalus wild plants and in vitro cultures produce different profiles of phenolic compounds with antioxidant activity, Food Chemistry 135(3): 1253-1260.

Dahmoune, F., Nayak, B., Moussi, K., Remini, H. and Madani, K. (2015). Optimization of microwave-assisted extraction of polyphenols from Myrtus communis L. leaves, Food Chemistry 166: 585-595.

Dandena, A., Zuka, L. and Kostova, M. (2014). Natural antioxidants in black chokeberry marc extracts depending on the extraction method., 9th Baltic Conference on Food Science and Technology "Food for Consumer Well-Being" FOODBALT 2014, Jelgava, Latvia, 8-9 May, 2014 pp. 324-326.

Deng, Y., Zhao, Y., Padilla-Zakour, O. and Yang, G. (2015). Polyphenols, antioxidant and antimicrobial activities of leaf and bark extracts of Solidago canadensis L., Industrial Crops and Products 74: 803-809.

Dent, M., Dragovic-Uzelac, V., Penic, M., Bosiljkov, T. and Levaj, B. (2013). The Effect of Extraction Solvents, Temperature and Time on the Composition and Mass Fraction of Polyphenols in Dalmatian Wild Sage (Salvia officinalis L.) Extracts, Food Technology and Biotechnology 51(1): 84-91.

Fecka, I. and Turek, S. (2008). Determination of polyphenolic compounds in commercial herbal drugs and spices from Lamiaceae: thyme, wild thyme and sweet marjoram by chromatographic techniques, Food Chemistry 108(3): 10391053

Galvan d'Alessandro, L., Kriaa, K., Nikov, I. and Dimitrov, K. (2012). Ultrasound assisted extraction of polyphenols from black chokeberry, Separation and Purification Technology 93: 42-47.

Horžic, D., Jambrak, A. R., Belščak-Cvitanovic, A., Komes, D. and Lelas, V. (2012). Comparison of Conventional and Ultrasound Assisted Extraction Techniques of Yellow Tea and Bioactive Composition of Obtained Extracts, Food and Bioprocess Technology 5(7): 2858-2870.

Hossain, M., Barry-Ryan, C., Martin-Diana, A. and Brunton, N. (2011). Optimisation of accelerated solvent extraction of 
antioxidant compounds from rosemary (Rosmarinus officinalis L.), marjoram (Origanum majorana L.) and oregano (Origanum vulgare L.) using response surface methodology, Food Chemistry 126(1): 339-346.

Jovanović, A. A., Đordevic, V. B., Zdunic, G. M., Pljevljakušic, D. S., Šavikin, K. P., Gođevac, D. M. and Bugarski, B. M. (2017). Optimization of the extraction process of polyphenols from Thymus serpyllum L. herb using maceration, heatand ultrasound-assisted techniques, Separation and Purification Technology 179: 369-380.

Khan, M. K., Abert-Vian, M., Fabiano-Tixier, A.-S., Dangles, O. and Chemat, F. (2010). Ultrasound-assisted extraction of polyphenols (flavanone glycosides) from orange (Citrus sinensis L.) peel, Food Chemistry 119(2): 851-858.

Lee, L.-S., Lee, N., Kim, Y., Lee, C.-H., Hong, S., Jeon, Y.-W. and Kim, Y.-E. (2013). Optimization of Ultrasonic Extraction of Phenolic Antioxidants from Green Tea Using Response Surface Methodology, Molecules 18(11): 13530-13545.

Milošević, S., Lepojević, Z., Zeković, Z. and Vidović, S. (2011). Determination of extraction conditions of Ginkgo biloba L. leaves by supercritical $\mathrm{CO} 2$ using response surface methodology, Hemijska industrija 65(2): 147-157.

Milutinović, M., Radovanović, N., Corović, M., Šiler Marinković, S., Rajilic-Stojanović, M. and DimitrijevićBranković, S. (2015). Optimisation of microwave-assisted extraction parameters for antioxidants from waste Achillea millefolium dust, Industrial Crops and Products 77: 333-341.

Miron, T., Plaza, M., Bahrim, G., Ibanez, E. and Herrero, M. (2011). Chemical composition of bioactive pressurized extracts of Romanian aromatic plants, Journal of Chromatography A 1218(30): 4918-4927.

Munin, A. and Edwards-Levy, F. (2011). Encapsulation of Natural Polyphenolic Compounds; a Review, Pharmaceutics 3(4): 793-829.

Mustafa, A. and Turner, C. (2011). Pressurized liquid extraction as a green approach in food and herbal plants extraction: A review, Analytica Chimica Acta 703(1): 8-18.

Nayak, B., Dahmoune, F., Moussi, K., Remini, H., Dairi, S., Aoun, O. and Khodir, M. (2015). Comparison of microwave, ultrasound and accelerated-assisted solvent extraction for recovery of polyphenols from Citrus sinensis peels, Food Chemistry 187: 507-516.

Oniszczuk, A. and Podgorski, R. (2015). Influence of different extraction methods on the quantification of selected flavonoids and phenolic acids from Tilia cordata inflorescence, Industrial Crops and Products 76: 509-514.

Pinelo, M., Fabbro, P., Manzocco, L., Nunez, M. and Nicoli, M. (2005). Optimization of continuous phenol extraction from byproducts, Food Chemistry 92(1): 109-117.

Pompeu, D., Silva, E. and Rogez, H. (2009). Optimisation of the solvent extraction of phenolic antioxidants from fruits of Euterpe oleracea using Response Surface Methodology, Bioresource Technology 100(23): 6076-6082.

Sen, S. and Chakraborty, R. (2011). The Role of Antioxidants in Human Health, in S. Andreescu and M. Hepel (eds), Oxidative Stress: Diagnostics, Prevention, and Therapy, Vol. 1083, American Chemical Society, Washington, DC, pp. 137. DOI: 10.1021/bk-2011-1083.ch001.
Simić, V. M., Rajković, K. M., Stojicević, S. S., Veličković, D. T. Nikolić, N. ., Lazić, M. L. and Karabegović, I. T. (2016). Optimization of microwave-assisted extraction of total polyphenolic compounds from chokeberries by response surface methodology and artificial neural network, Separation and Purification Technology 160: 89-97.

Ćujic, N., Šavikin, K., Jankovic, T., Pljevljakušic, D., Zdunic, G. and Ibric, S. (2016). Optimization of polyphenols extraction from dried chokeberry using maceration as traditional technique, Food Chemistry 194: 135-142.

Uma, D. B., Ho, C. W. and Aida, W. M. W. (2010). Optimization of extraction parameters of total phenolic compounds from henna (Lawsonia inermis) leaves, Sains Malaysiana 39(1): 119-128.

Vajić, U.-J., Grujić-Milanović, J., Živković, J., Šavikin, K., Gođevac, D., Miloradović, Z., Bugarski, B. and MihailovićStanojević, N. (2015). Optimization of extraction of stinging nettle leaf phenolic compounds using response surface methodology, Industrial Crops and Products 74: 912-917.

Vergara-Salinas, J. R., Perez-Jimenez, J., Torres, J. L., Agosin, E. and Perez-Correa, J. R. (2012). Effects of Temperature and Time on Polyphenolic Content and Antioxidant Activity in the Pressurized Hot Water Extraction of Deodorized Thyme (Thymus vulgaris), Journal of Agricultural and Food Chemistry 60(44): 10920-10929.

Vuleta, G., Milic, J. and Savic, S. (2012). Farmaceutska tehnologija (Pharmaceutical technology), Faculty of Pharmacy, University of Belgrade, Belgrade, Serbia.

Wang, L. and Weller, C. L. (2006). Recent advances in extraction of nutraceuticals from plants, Trends in Food Science $\mathcal{E}$ Technology 17(6): 300-312.

Wong Paz, J. E., Muniz Marquez, D. B., Martinez Avila, G. C., Belmares Cerda, R. E. and Aguilar, C. N. (2015). Ultrasoundassisted extraction of polyphenols from native plants in the Mexican desert, Ultrasonics Sonochemistry 22: 474-481.

Yang, Y. and Zhang, F. (2008). Ultrasound-assisted extraction of rutin and quercetin from Euonymus alatus (Thunb.) Sieb, Ultrasonics Sonochemistry 15(4): 308-313. 